\title{
Prerequisites for Phase I and II Clinical Drug Trials in Human
}

\author{
Michel Bourin ${ }^{1 *}$ and Abdeslam Chagraoui ${ }^{2}$ \\ ${ }^{1}$ Neurobiology of Mood disorders, University of Nantes, 98 rue Joseph Blanchart 44100 Nantes, France. \\ ${ }^{2}$ Inserm U982, Laboratory of Neuronal and Neuroendocrine Differentiation and Communication, Institute for Research and Innovation in Biomedicine, \\ Normandy University, France; Department of Medical Biochemistry, Rouen University Hospital, Rouen, France
}

Received: May 29, 2016; Accepted: June 01, 2016; Published: June 20, 2016

*Corresponding author: Michel Bourin, Neurobiology of Mood disorders, University of Nantes, 98 rue Joseph Blanchart 44100 Nantes, France

\begin{abstract}
The essential purpose of this paper is to consider in detail the knowledge of the prerequisites for drug studies in humans. In fact, the transition from the animal to man is always difficult, and very strict regulations have been established so that phase I trials (the first level of studies in man) can be performed in conditions ensuring the greatest safety for the individuals concerned.
\end{abstract}

Keywords: Analytic file; Galenic file; Pharmacodynamics file; Toxicologic file

\section{Introduction}

Regardless of the pharmacological class of the drugs used in clinical trials before their approval and registration, certain prerequisites are essential before the start of phases I and II. These prerequisites have been considered in different directives of the European Community Council which have appeared in the Official Journal of the European Community and in a work on toxicology. The purpose of the present document was to present a summary of these recommendations, notably concerning non-clinical requirements, the pharmaceutical quality of the investigational medical product and the conditions under which this type of clinical trial may be performed [1].

Five types of files concerning the new drug should be made available to investigators before the start of phase I and II clinical trials: the analytic file, the galenic file, the file on animal pharmacology, the pharmacodynamic file and the toxicologic file. The objective is to answer precise questions which condition the continuation or suspension of the drug's development program. Exploratory trials include a small number of patients or healthy subjects and expose them (over a short period of time) to a low dose of an investigational medicinal product

According to the Guide to Good Clinical Practices for drug trials, these files are prepared under the supervision of the manufacturer promoting the trials who is responsible for communicating them in their entirety to the investigators. However, the investigators must solicit this information from the manufacturer and be fully informed before the final phase of drafting the protocol-and necessarily before the start of the clinical trials. This information (or a detailed summary) must also be communicated to the Advisory Committee for the Protection of Biomedical Research Subjects (a state-authorized ethics committee) which gives its opinion on proposed protocols.

\section{Analytic and galenic files}

These two files describe the characteristics of the new drug proposed for clinical trials.The analytic file provides a schema of the chemical formula of the drug, a description of the physicochemical properties of the substance as well as its purity and stability conditions, and various details concerning solubility, particle size, hydration state, the oil/water partition coefficient, $\mathrm{pK} / \mathrm{pH}$ values, etc.

The galenic file contains the complete description of the galenic (or pharmaceutic) form(s) of the drug prepared for the clinical trials: galenic form (capsule, tablet, solution, etc.), composition in active principle and excipient, stability period, possible sterilization conditions, special precautions for conservation, compatibility with injectable solvents, nature and content of the recipient, etc. The labeling conditions for samples are also indicated.

These files, which are prepared by the pharmacists in charge of pharmaceutical laboratories, describe in detail the characteristics of the drug to be used during phase I and II clinical trials. As it often happens that various galenic forms of the same drug are produced and tested in man before the choice of the definitive galenic form(s), it is important to specify clearly for each clinical trial performed the galenic form and the batch number of the drug which has been tested. As the bioavailability of a drug can vary from one galenic form to another, bioequivalence studies among the various galenic forms should be planned if the form is modified during the trials (e.g. the administration of an aqueous form of the drug for phase I trials and the use of capsules or tablets in phase II trials). However, these opportunities for rapid entry into the clinic must be balanced by a consideration of the unique risks associated with first-in-human clinical trials, and by accounting for public concerns regarding drug safety in general. This feature review discusses how innovative clinical strategies can be used effectively in early drug development.It would of course be desirable to have the definitive galenic form of the 
drug available as soon as possible for the clinical trials. However, this is rarely the case since the choice of the definitive form often depends on the results of the first clinical trials.

\section{Animal pharmacokinetic file}

Pharmacokinetic studies in animals are performed on several species of rodents (most often the rat) or larger mammals (the dog) and can include studies in primates (monkeys).The purpose of these studies is to describe the following parameters: absorption and bioavailability of the drug, distribution of the drug in the organism and its binding to plasma proteins, and elimination of the drug.

The study of the metabolism of the drug in animals reveals the main metabolic pathways, the possible existence of circulating metabolites, and their main elimination route. The isolation of these metabolites, the characterization of their chemical structure, and the study of their possible pharmacologic activity should be performed as soon as possible in order to determine whether circulating metabolites should be taken into account in pharmacologic and toxicologic studies. Moreover, these initial kinetic studies in the animal will allow the detection of a possible accumulation of the parent product (and/or its metabolites), which would require special precautions during preclinical studies and the first human trials. The possibility of using substances labeled with radioactive isotopes (H3, C14) without limitations in the animal greatly facilitates these studies, allowing rapid performance of evaluative studies (absorption and elimination of the drug) as well as studies of drug distribution in the animal organism.

Pharmacokinetic studies in the animal are of considerable interest because of their value in predicting human kinetics. However, the importance of possible differences between species should not be overlooked. The dog, for example, is a slow acetylator and will synthesize few acetylated metabolites (if this metabolic pathway is involved). The interpretation of the results of these studies must thus remain subject to criticism and should always be compared with results for the human kinetics of the drug. In particular, preliminary kinetic studies in man will allow the choice of "the species closest to (or least remote from) man" in order to provide more thorough pharmacokinetic investigations.

"Recommendation 83/571/EEC introduced for the first time the concept of studying pharmacokinetics and metabolism in animals in terms of safety." The purpose of such studies is to determine the concentrations of the drug and its metabolites, as well as their kinetics in blood, biological fluids and organs; obtain information on the relations between toxicity and drug concentrations; study the possibility of enzymatic induction and of drug accumulation in case of repeated administration; and select (insofar as possible) the animal species to be used for toxicologic studies on the basis of a similarity with the human situation regarding the fate of the drug in the organism.

Thus, these investigations may require a preliminary study of the kinetics and metabolism of the drug in a limited number of human subjects before a definitive choice can be made of the animal species to be used for toxicity studies involving repeated administrations.

\section{Pharmacodynamic file}

This file is obviously of major importance to investigating physicians insofar as it contains the description of all the known pharmacodynamic properties of the drug. Major new developments in pharmaceutical technology which have produced controlled-release delivery systems and the market introduction of generics would never have occurred but for the active contribution of pharmacokinetics [2].

Pharmacodynamics relative to the main property The main pharmacologic property, which relates to theintended therapeutic use of the drug, has generally been studied and determined in several types of preparations and experimental models: in molecular, subcellular, cellular and tissue pharmacology when the drug acts on individualized receptors in vitro; in isolated and perfused organs; and in animal models in vivo (anesthetized and conscious animals), which reproduce as closely as possible the human pathological situation corresponding to the intended threapeutic use of the drug (relations between dose and effect and plasma concentration and effect are determined in animals, sometimes allowing a modeling of the situation for comparison with results obtained in human subjects in phase I and II trials).These studies in animals also permit an approach to the mechanism of drug action, although the physiopathology of the animal models must not differ too greatly from that of the pathologic clinical situation.

Finally, pharmacologic studies in animals, by comparing the properties of the new drug with those of reference products of the same therapeutic class, allow the relative potency of the drug to be studied and to situate the new substance with respect to existing compounds used in therapy [3].

General Pharmacodynamics The effects of the new drug on all important functions of the organism are studied in animals. Thus, cardiovascular, renal, pulmonary, neurologic, sensory, gastrointestinal, endocrine and metabolic properties are considered either in pharmacodynamic studies or during toxicologic studies by comparing acute and repeated administrations of the drug. Other properties, such as the effects on different experimental inflammation models or of the drug on gastric mucosa, are studied more specifically.

\section{Toxicologic file}

The toxicologic file includes six types of studies:

1. Acute toxicity or toxicity by a single administrationThis study permits the calculation of the dose (and corresponding administration route) inducing the death of the animal. The minimum lethal dose (MLD) by slow intravenous infusion permits the choice of the doses to be used for determining the median lethal dose (LD50).

LD50, i.e. the dose capable of killing half of the experimental animals by the chosen administration route, is determined by suitable methods. It is calculated in two animal species (most often the mouse and rat) using an equal number of animals of each sex and at least two administration routes.

2. Toxicity by repeated administration for limited periods 
To avoid a delay until the end of the long-term toxicity studies (1 year) that precede clinical trials, the European Union has proposed minimum periods for toxicity studies before initial administrations of the drug to human subjects (Table 1).

3. "Chronic" toxicity When the drug is intended for long-term administration in man, toxicity studies should be performed over a one- or even two-year period, which corresponds to a large part of the life of a laboratory animal. These studies are of particular interest if the following conditions are satisfied:

- Choosing an animal species as close as possible to man with respect to drug kinetics and metabolism.

- Choosing dosage and administration routes that allow undesirable effects to be induced (in practice, three concentrations are required: a non-toxic one, a highly toxic one leading to the death of a certain percentage of animals, and an intermediary one). These toxicologic studies should include one using the same drug administration route as that proposed for clinical trials.

- Requiring that the target organ and the active principle be the same as those involved in the human therapeutic effect.

- Collecting as many data as possible on the experimental animals (mortality, morbidity, behavior, weight, physiological and biological modifications, histopathological analysis of 31 tissues or organs, etc.).

- Checking the effects of animal exposure to drugs (toxicokinetics) by measurement of plasma concentrations and comparison with human data.

The pharmacologic data and the correlations between plasma concentrations and effects observed during these studies can be of considerable predictive value for the subsequent clinical trials.

4. Determination of mutagenic potency This study concerns the potency of a substance in inducing a modification in genetic material by acting on DNA. In practice, a mutagenic test is conducted on bacteria as well as by means of a chromosomal mutation test. However, many other tests can also be performed, particularly to determine metabolic activation.

\section{Determination of effects on reproductive functions}

Studies of the effects of new drugs on reproductive functions require three phases or segments:

Segment 1: a fertility study.

Segment 2: studies of embryotoxicity, fetotoxicity and teratogenic potency.

Segment 3: study of peri- and postnatality. Because of the length of these studies, phase I and II clinical trials may be initiated without waiting for the results of reproduction studies, provided that:

1. Women are excluded from the trials,
2. Repeated toxicity studies have shown nogonadal anomalies,

3. The drug is not part of a "suspect" chemical series with a history of known toxic effects on reproductive functions.

\section{Determination of carcinogenic potency}

These investigations also involve long-term studies (18 months in the mouse and hamster and 24 months in the rat) which are only required if prolonged treatment is planned in man or if frequent intermittent administration of the drug to human subjects leads to prolonged exposure. Phase I and II clinical trials can be initiated without waiting for the results of carcinogenic studies, provided that:

1. Mutagenic tests have shown no anomalies,

2. The drug and/or its metabolites do not accumulate in the organism,

3. The drug is not part of a "suspect" chemical series with a history of animal or clinical epidemiological observations indicative of an iatrogenic carcinogenic risk.

\section{Conclusion}

Exploratory trials are clinical trials performed early in Phase I, prior to dose escalation and safety and tolerability trials [4]. These trials are de facto first-in-man studies but lack a therapeutic or diagnostic goal and do not seek to establish the maximum tolerated dose (MTD).This short presentation of prerequisites is intended as a reminder to investigating physicians of the need to ensure the safety of subjects and patients included in phase I and II trials respectively. Moreover, a thorough consideration of these prerequisites is often decisive in orienting the first clinical trials of the drug, indicating the specific parameters to be considered and guiding the first evaluations of the drug in man. Exploratory clinical trials provide a strategy for rapid human entry of investigational drugs. Such clinical studies are typically conducted during early clinical development in phase I as firstin-human studies, have no therapeutic intent, are not intended to examine clinical tolerability and involve a small number of human subjects at limited dose/exposure. Early decision data derived from such clinical studies may include PK, PD and/or biomarker-based translational medicine endpoints as well as PK/PD modeling approaches.

\section{References}

1. Francillon A, Pickering G, Belorgey C. Exploratory clinical trials: implementation modes \& guidelines, scope and regulatory framework. 2009;64(3):149-159. doi: 10.2515/therapie/2009022.

2. Marzo A. Clinical pharmacokinetic registration file for NDA and ANDA procedures. Pharmacol Res. 1997;36(6):425-450.

3. Muller PY. Comparative requirements for exploratory clinical trials -- eIND, eCTA and microdosing. Adv Drug Deliv Rev. 2011;63(7):511517. doi: 10.1016/j.addr.2010.10.010.

4. Bourin M. Clinical drug trials in psychiatry. Formation of the investigator. Amazon Kindle. 2016;186. 